\title{
Self-Induced Torque in Hyperbolic Metamaterials
}

\author{
Pavel Ginzburg, ${ }^{1, *}$ Alexey V. Krasavin, ${ }^{1}$ Alexander N. Poddubny, ${ }^{2,3}$ Pavel A. Belov, ${ }^{2}$ \\ Yuri S. Kivshar, ${ }^{2,4}$ and Anatoly V. Zayats ${ }^{1}$ \\ ${ }^{1}$ Department of Physics, King's College London, Strand, London WC2R 2LS, United Kingdom \\ ${ }^{2}$ National Research University for Information Technology, Mechanics and Optics (ITMO), St. Petersburg 197101, Russia \\ ${ }^{3}$ Ioffe Physical-Technical Institute of the Russian Academy of Sciences, St. Petersburg 194021, Russia \\ ${ }^{4}$ Nonlinear Physics Center and Center for Ultrahigh Bandwidth Devices for Optical Systems (CUDOS), \\ Research School of Physics and Engineering, Australian National University, Canberra ACT 0200, Australia
}

(Received 21 December 2012; revised manuscript received 5 April 2013; published 19 July 2013)

\begin{abstract}
Optical forces constitute a fundamental phenomenon important in various fields of science, from astronomy to biology. Generally, intense external radiation sources are required to achieve measurable effects suitable for applications. Here we demonstrate that quantum emitters placed in a homogeneous anisotropic medium induce self-torques, aligning themselves in the well-defined direction determined by an anisotropy, in order to maximize their radiation efficiency. We develop a universal quantummechanical theory of self-induced torques acting on an emitter placed in a material environment. The theoretical framework is based on the radiation reaction approach utilizing the rigorous Langevin local quantization of electromagnetic excitations. We show more than 2 orders of magnitude enhancement of the self-torque by an anisotropic metamaterial with hyperbolic dispersion, having negative ratio of permittivity tensor components, in comparison with conventional anisotropic crystals with the highest naturally available anisotropy.
\end{abstract}

PACS numbers: 73.20.Mf, 42.50.-p, 81.05.Xj

The first hypothesis on light induced forces was seemingly done by Kepler, who tried to attribute the behavior of comet (gaseous) tails directed always away from the Sun due to the solar breeze [1]. The term "radiation pressure," i.e., force acting on an uncharged body situated in an electromagnetic field, was introduced by Maxwell, and the first experimental evidence of the effect was reported in 1901 [2]. The real boost of practical applications was initiated with the discovery of optical tweezers [3]. This has started the experiments based on precise positioning and trapping of micro- and nanosized objects, such as small biological species [4], and even studies of conformation events at the molecular level [5]. The concept of optical forces has been employed in laser cooling (or radiation pressure cooling) [6], precise particles' sorting [7], artificial light crystals (optical lattices) [8], quantum computing [9], solar sails [10], and many other areas.

One of the best known examples of the self-induced forces is the Abraham-Lorentz or radiation reaction force resulting in self-induced damping produced by accelerating particle on itself and accompanied by far-field radiation. The introduction of this force enables calculations of spontaneous emission rate without the procedure of second quantization [11], while full classical-quantum correspondence may also be shown [12].

Typical optical forces achievable with reasonable light intensities are, however, in the $10^{-9}-10^{-12} \mathrm{~N}$ range, which imposes certain limitation on the size and weight of the objects that can be manipulated due to their stochastic interactions with surrounding, e.g., Brownian motion and viscosity of a surrounding medium, if present. One of the very promising and already tested approaches for the optical force enhancement relies on the increase of the field gradient with the help of plasmonic nanostructures [13]. Metamaterials with negative refractive index have also a striking effect on radiation pressure, reversing its direction, so that negative index bodies are attracted by light beam $[14,15]$.

In this Letter we have developed a rigorous quantummechanical theory of the self-induced torque acting on a dipole situated in a structured material environment with arbitrary absorption, dispersion, and spatial variations. The theoretical framework is based on the radiation reaction approach and the rigorous Langevin local quantization of electromagnetic excitations in coordinate domain. We have shown that the induced torque does not necessarily depend on the reflected waves from material boundaries and can emerge in spatially homogeneous structures. We have investigated this effect on the example of a radiating dipole, e.g., a chemically excited polar molecule, situated in anisotropic but homogeneous material. We have further demonstrated that a dipole situated within hyperbolic anisotropic metamaterial exhibits a giant self-induced torque and aligns itself in the direction perpendicular to the negative component of the permittivity tensor. The magnitude of the torque is orders of magnitude larger than in any other anisotropic materials existing in nature. A realistic (not homogeneous) hyperbolic metamaterial configuration has also been considered revealing a similar effect on a radiating dipole.

In order to derive radiation reaction forces from the equation of motion, we consider a one dimensional (1D) 
case of a dipole in an external electromagnetic field described by the Hamiltonian [12]

$\hat{H}=\frac{\hat{\vec{p}}^{2}}{2 m}-\int d^{3} \vec{r} \vec{\mu}(\vec{r}) \cdot \vec{E}(\vec{r}, t)+\frac{1}{2} m \omega_{0}^{2} \tilde{x}^{2}+\hat{H}_{\text {field }}$,

where $\hat{\vec{p}}$ is the momentum operator of the particle, $m$ is its mass, $\vec{\mu}(\vec{r})$ is its moment density, $\omega_{0}$ is the undamped oscillator angular frequency, $E$ is the external electric field, while $\hat{H}_{\text {field }}$ is the total energy of the field. The coordinate $\tilde{x}$ describes the dipole's internal dynamics, while the position of the quantum system in space is described by the global coordinate domain $\vec{r}$. The interaction Hamiltonian $\hat{H}_{\text {int }}=-\int d^{3} \vec{r} \vec{\mu}(\vec{r}) \cdot \vec{E}(\vec{r}, t)$ takes into account the spatial nonlocality of the dipole wave functions by introducing the integration over the electron density defined as $\vec{\mu}(\vec{r})=e\left[\psi_{e}^{*}(\tilde{x}) \psi_{g}(\tilde{x})\right] \delta(\tilde{y}) \delta(\tilde{z}) \hat{\tilde{x}}$ in the 1D system under consideration and $\hat{\tilde{x}}$ is the unit vector in the internal coordinate system. The initial state of the dipole is the first excited state $\left[\psi_{e}(\tilde{x})\right]$ of the harmonic oscillator which then spontaneously decays to the ground state $\left[\psi_{g}(\tilde{x})\right]$, obtaining recoil (both translational and rotational) during the decay process.

The next step requires the quantization of the electromagnetic field involved in the interaction. If the oscillator is situated in a free space, the straightforward "plane wave" quantization may be used, and the quantum expression for the force may be obtained with the correspondence to the classical expression [12]. However, the direct field quantization based on the field expansion over the classical electromagnetic modes of a system, known as mode decomposition, is not applicable for lossy systems [16]. The rigorous field quantization technique for dispersive and lossy systems could be based on incorporation of material degrees of freedom [17] or on the introduction of local Langevin noise operators [18]. In the latter approach, the field operators are strictly related to the classical electromagnetic Green's functions, and the dispersion and losses can be included without violating canonical commutation rules. Using this formalism, the positive frequency part of the electromagnetic field operator can be written as [19]

$$
\begin{aligned}
\hat{\vec{E}}^{(-)}(\vec{r}, \omega, t)= & i \sqrt{\frac{\hbar}{\pi \varepsilon_{0}}} \int_{0}^{\infty} d \omega \cdot\left(\frac{\omega}{c}\right)^{2} \int d^{3} \vec{s} \\
& \cdot \sqrt{\operatorname{Im} \varepsilon(\vec{s}, \omega)} \overleftrightarrow{G}(\vec{r}, \vec{s}, \omega) \hat{\vec{f}}(\vec{s}, \omega, t) .
\end{aligned}
$$

Here, $\hat{\vec{f}}(\vec{r}, \omega)$ is the local annihilation field operator, $\overleftrightarrow{G}\left(\vec{r}_{A}, \vec{r}, \omega\right)$ is the classical electromagnetic Green's tensor defined by $\left[\nabla \times \nabla \times-(\omega / c)^{2} \varepsilon(\vec{r}, \omega)\right] \overleftrightarrow{G}\left(\vec{r}, \vec{r}_{A}, \omega\right)=$ $\delta\left(\vec{r}-\vec{r}_{A}\right)$, where $\vec{r}_{A}$ is the source position, $\varepsilon_{0}$ is the vacuum permittivity, and $\varepsilon(\vec{r}, \omega)$ is the position and frequency dependent relative material permittivity.

Solving the Heisenberg equations of motion for the field operators $i \hbar d \hat{f}_{\lambda} / d t=\left[\hat{f}_{\lambda}, H\right]$ and separating the oscillatorrelated part from the free space contribution, we obtain the generalized expression of the radiation reaction force density $\vec{f}_{r r}(\vec{r})$,

$$
\begin{aligned}
\vec{f}_{r r}(\vec{r})= & \frac{i e}{\pi \varepsilon_{0}} \int d^{3} \vec{r}^{\prime}\left[\psi_{e}^{*}(\vec{r}) \psi_{g}(\vec{r})\right]\left\{\int_{0}^{\infty} d \omega\left(\frac{\omega}{c}\right)^{2}\right. \\
& \left.\times \int_{0}^{t} d t^{\prime} \vec{\mu}\left(\vec{r}^{\prime}, t^{\prime}\right) e^{i \omega\left(t^{\prime}-t\right)} \operatorname{Im} \overleftrightarrow{G}\left(\vec{r}, \vec{r}^{\prime}, \omega\right)\right\}+ \text { c.c. }
\end{aligned}
$$

where c.c. stands for complex conjugate. The force density is introduced here instead of the usual radiation reaction force in order to preserve the spatial nonlocality of the oscillator.

With the help of the above formalism, it is also possible to obtain the spontaneous emission rate and the Lamb shift of the oscillator levels. Defining the work performed by the radiation reaction on the oscillator, we attribute its imaginary part to the radiative decay rate $\gamma$, while the real part will determine the Lamb shift $\Delta \omega$,

$$
\begin{gathered}
\gamma=\frac{2 \omega_{0}^{2}}{\varepsilon_{0} \hbar c^{2}} \operatorname{Im}\left\{\iint d^{3} \vec{r} d^{3} \vec{r}^{\prime} \vec{\mu}(\vec{r}) \overleftrightarrow{G}\left(\vec{r}, \vec{r}^{\prime}, \omega_{0}\right) \vec{\mu}\left(\vec{r}^{\prime}\right)\right\}, \\
\Delta \omega=-\frac{\bar{\omega}_{0}^{2}}{\varepsilon_{0} \hbar c^{2}} \operatorname{Re}\left\{\iint d^{3} \vec{r} d^{3} \vec{r}^{\prime} \vec{\mu}(\vec{r}) \overleftrightarrow{G}\left(\vec{r}, \vec{r}^{\prime}, \bar{\omega}_{0}\right) \vec{\mu}\left(\vec{r}^{\prime}\right)\right\} .
\end{gathered}
$$

The well-known expressions for these parameters [18] coincide with Eq. (4) if the spatial nonlocality of the emitter wave function is ignored. Such ignorance will, however, cause divergence in $\Delta \omega$, since the real part of the electromagnetic Green function diverges when $\vec{r}=\vec{r}^{\prime}$, and requires additional treatment.

It is quite remarkable that the radiation reaction force, having a vectorial nature, is not always aligned in the direction of the emitting dipole, giving rise to the selfinduced torque density described by $\vec{t}(\vec{r})=\vec{r} \times \vec{f}_{r r}(\vec{r})$. The measurable value in the laboratory frame is obtained by the time averaging of the expectation value of the integrated density,

$$
\begin{aligned}
T & =\langle\vec{T}\rangle_{\text {time }} \\
& =\frac{\omega_{0}^{2}}{\varepsilon_{0} c^{2}} \operatorname{Re}\left\{\iint d^{3} \vec{r} d^{3} \vec{r}^{\prime} \vec{\mu}^{*}\left(\vec{r}^{\prime}\right) \times \overleftrightarrow{G}\left(\vec{r}, \vec{r}^{\prime}, \omega_{0}\right) \vec{\mu}\left(\vec{r}^{\prime}\right)\right\}
\end{aligned}
$$

Thus, the torque is proportional to the dipole-induced electromagnetic field in the surrounding space vectorially multiplied by the dipole moment. The mathematical singularity of the self-induced torque is removed by considering the spatially distributed electron wave function, typically several nanometers for solid state emitters [20].

To illustrate the environmentally induced torques on a radiating system, we first consider a point dipole situated above a perfectly conducting surface. The dipole is tilted at the angle $\alpha$ with respect to the surface normal and placed at the distance $d$ from the surface [Fig. 1(a)]. For distances $d \ll \lambda$, the torque on the dipole is 

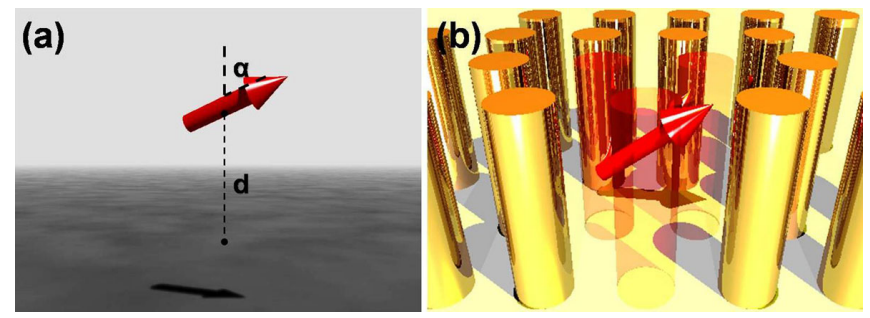

FIG. 1 (color online). Schematics of the radiating dipole situated above a perfect mirror and inside a hyperbolic material realized as an infinite array of vertically aligned plasmonic nanorods.

$$
T=\frac{\vec{\mu}^{2} \sin 2 \alpha}{32 \pi \varepsilon_{0} d^{3}} .
$$

This torque leads to the preferential direction of the dipole orientation perpendicular to the surface in order to maximize its radiation efficiency resulting in the minimum potential energy in the near field. This simple case emphasizes the feedback induced by environment on a radiating system.

Structured materials can support more complex (than a reflection from a mirror) Greens functions that may result in strong self-induced torques. One of the interesting examples is hyperbolic metamaterials-one- or twodimensional composites [21,22] described as an effective anisotropic medium with hyperbolic isofrequency surfaces in certain frequency ranges. Such metamaterials have different signs of the longitudinal $\left(\varepsilon_{\|}\right)$and transverse $\left(\varepsilon_{\perp}\right)$ components of the permittivity tensor [Fig. 1(b)]. In the following, we show the giant self-induced torque enhancement in hyperbolic metamaterial in comparison to natural birefringent crystals.

For quantitative estimation of the dipole self-action given by Eq. (5), we have employed finite element electromagnetic simulations, assuming the Gaussian distribution of the dipole density in an effectively two-dimensional anisotropic medium. This density $\vec{\mu}(\tilde{x})=\vec{\mu}_{0} e^{-\left(\tilde{x}^{2} / w^{2}\right)}$, where $w$ is the distribution width and $\mu_{0}$ is proportional to the overall dipole moment, has been discretized into a line of individual dipoles. The line discretization corresponds to the one-dimensional model given by the Hamiltonian in Eq. (1). Each individual dipole in the discretization experiences a torque induced by all the rest (but not by itself), and the total force on the dipole is calculated as a sum over the density distribution. Equation (5) contains the emission pattern of a dipole in the medium, which plays a key role in the resulting summation. These dipole radiation patterns (the electric field amplitude) in various anisotropic media are shown in Fig. 2. The left column corresponds to the material with $300 \%$ conventional, elliptic, anisotropy $\left(\varepsilon_{\perp}=1, \varepsilon_{\|}=3\right)$ and the middle column to the homogeneous metamaterial with hyperbolic dispersion $\left(\varepsilon_{\perp}=-1-0.2 i, \varepsilon_{\|}=3-0.2 i\right)$.

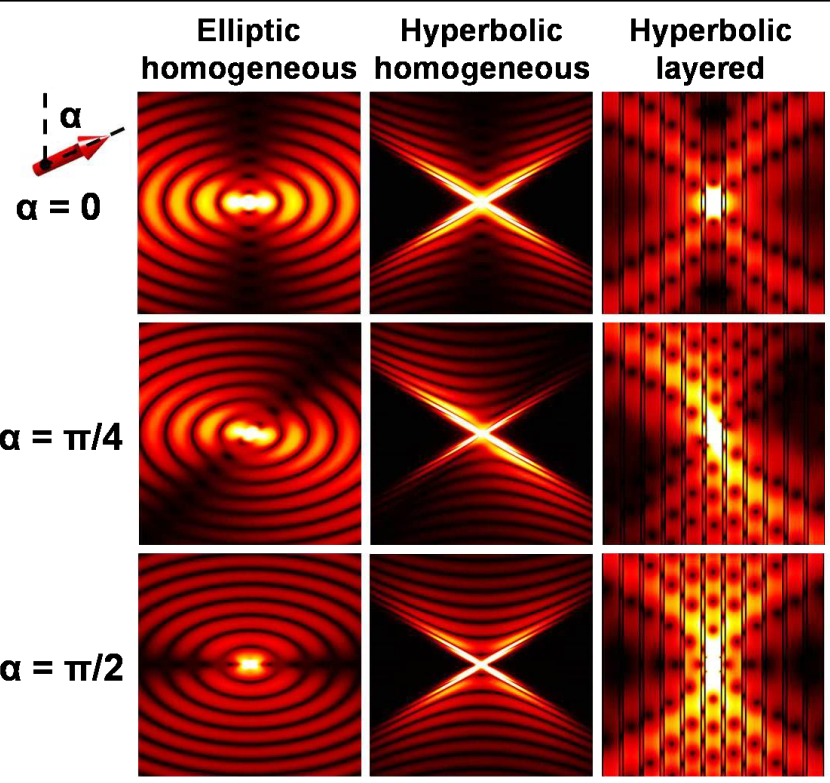

FIG. 2 (color online). Radiation patterns $(|\operatorname{Re} \vec{E}|)$ of a dipole emitter in anisotropic medium: (Left) conventional (elliptic) anisotropic medium with $\varepsilon_{\perp}=1, \varepsilon_{\|}=3$, (middle) hyperbolic medium with $\varepsilon_{\perp}=-1, \varepsilon_{\|}=3$, (right) layered metamaterial realization of the hyperbolic medium. The dipole orientation angle is measured with respect to the $\varepsilon_{\perp}$ direction as indicated. The color scale is the same for all the plots.

The emission patterns correspond to different tilting angles $\alpha$ of the dipole with respect to the extraordinary $(\perp)$ axis. While the radiation pattern of the dipole in positively anisotropic medium is rather uniform, the hyperbolic metamaterial results in highly localized, directional emission which is responsible for strong self-induced torques.

For quantitative estimation of the self-induced torque, a dipole with the finite size and corresponding to the Gaussian half-width of $2 \mathrm{~nm}$ (e.g., rhodamine-like molecules having dipole moments of the order of several Debye) was considered. We have first considered a positive anisotropy of only $10 \%\left(\varepsilon_{\perp}=2.43, \varepsilon_{\|}=3\right)$, which approximately correspond to a rutile $\left(\mathrm{TiO}_{2}\right)$ crystal. The calculated torque is zero when the dipole is aligned along the crystallographic directions of the material and has a broad maximum for the orientation not coinciding with the lattice directions (Fig. 3). With the increase of the positive anisotropy to $300 \%\left(\varepsilon_{\perp}=1, \varepsilon_{\|}=3\right.$, not possible with any existing natural materials), the torque increases by about 1 order of magnitude. In both cases, the orientation $\alpha=90^{\circ}$ is unstable, since the angular derivative is positive, while orientation $\alpha=0^{\circ}$ is stable equilibrium, so that the dipole will align itself in the direction of the lowest tensor component. The dipole rotation in a positively anisotropic medium can also be described in the static limit by the solution of the appropriate Poisson equation, giving the torque dependence in the $2 \mathrm{D}$ case as 


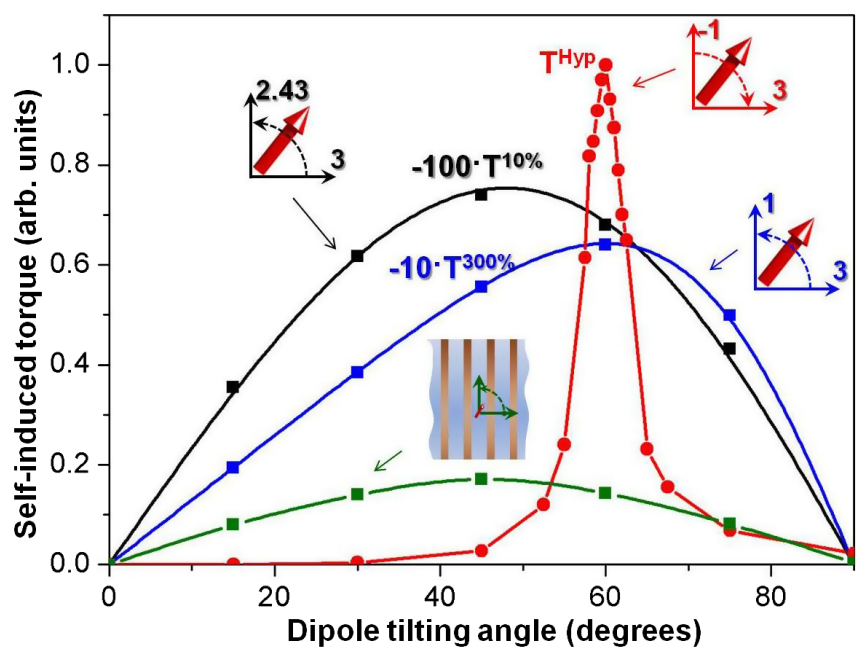

FIG. 3 (color online). Torque on a $2 \mathrm{~nm}$ size dipole situated in the anisotropic medium as a function of its tilting angle with respect to the extraordinary axis: (black squares) $\varepsilon_{\perp}=2.42$, $\varepsilon_{\|}=3$, (blue squares) $\varepsilon_{\perp}=1, \varepsilon_{\|}=3$, (red circles) $\varepsilon_{\perp}=-1$, $\varepsilon_{\|}=3$, (green squares) layered metamaterial with effective parameters $\varepsilon_{\perp}=-1-0.2 i, \varepsilon_{\|}=2.9$, not scaled. Black and blue lines are the analytical fits, red and green lines are guides for the eye. The rotation directions are indicated in the insets.

$$
T \sim \frac{\left(\varepsilon_{\perp}-\varepsilon_{\|}\right) \sin 2 \alpha}{\sqrt{\varepsilon_{\perp} / \varepsilon_{\|}}\left(\varepsilon_{\|} \varepsilon_{\perp} \sin ^{2} \alpha+\varepsilon_{\perp}^{2} \cos ^{2} \alpha\right)} .
$$

Equation (7) was used to fit the angular dependence of the numerical data (Fig. 3). The correspondence between the numerical points and analytical static fit shows, in particular, that our procedure of the singularity removal is the valid numerical approach. Moreover, it may be analytically shown that substitution of a one-dimensional charge distribution along the dipole line into Eq. (5) leads to Eq. (7). On the other hand, Eq. (7) is not applicable for the hyperbolic anisotropy (e.g., torques will be complex numbers), highlighting the demand for the developed above quantum description.

In comparison to the positive anisotropy case, the torque on the dipole placed in hyperbolic metamaterial $\left(\varepsilon_{\|}=3\right.$, $\varepsilon_{\perp}=-1$ ) has a much sharper peak (Fig. 3), originating from the more pronounced directionality of the dipole radiation pattern in hyperbolic medium (Fig. 2). The analysis shows that the equilibrium orientation of the dipole will correspond to the direction along the largest (positive) component of the dielectric tensor, in contrast to a conventional anisotropy case, and the overall torque is enhanced by almost 1 order of magnitude compared to the conventional material with the same absolute ratio of ordinary and extraordinary permittivities. Thus, this enhancement can be distinctively attributed to the hyperbolic dispersion of the metamaterial, and not just to the large ratio of the tensor components.

One of the significant questions on the way towards practical observation of the effect is the realization of the hyperbolic metamaterial. The finite size of the unit cell will introduce certain limitations on the validity of the consideration of a dipole within the homogenization (effective medium) model. Qualitatively, the electromagnetic waves with wave vectors of order of the inverse period of the structure will be affected by the granularity of the composite. On the other hand, the emitter dimension will define the range of the radiated wave vectors implying two different regimes: extended (bigger than a unit cell) and localized (smaller than it) emitter. While the "extended emitter" could be treated within the homogeneous material model, the "localized" one requires extra care. In the latter case, the fields over the emitter dimensions will be homogeneous, yet anisotropic. As a result, the angular dependence of the torque will be similar to those of Eq. (6). For comparison with the effective medium model, we have studied a layered hyperbolic metamaterial realized as a periodic silica $(22 \mathrm{~nm}) /$ gold $(5 \mathrm{~nm})$ stack with the effective permittivity tensor components $\varepsilon_{\perp}=-1-0.2 i$ and $\varepsilon_{\|}=2.9$ at the $700 \mathrm{~nm}$ emission wavelength, similar to the previously considered homogeneous hyperbolic material. Radiation patterns of a dipole within layered material (Fig. 2) show similar behavior to the homogeneous case but with a much broadened directionality of emission. This in turn leads to the significant reduction of the torque (Fig. 3), but the fundamental phenomenon of the rotation is clearly preserved.

Finally, to represent the concept of the radiation efficiency maximization, corresponding to the minimum near-field energy at the equilibrium position of the dipole, we have calculated the orientation-dependent energy flux through the virtual far-field sphere of $2 \mu \mathrm{m}$ radius, inclosing the radiation source (Fig. 4). For the elliptic anisotropy, the energy flux reaches maximum when $\alpha=0^{\circ}$, while the

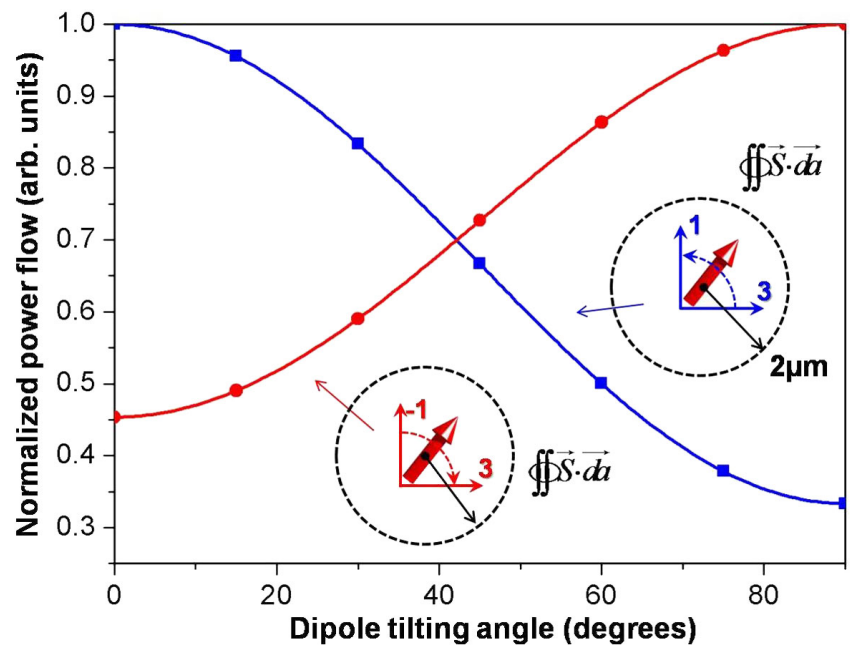

FIG. 4 (color online). Normalized far-field power flow through the virtual $2 \mu \mathrm{m}$ radius sphere as the function of the dipole orientation angle: (blue) elliptic and (red) hyperbolic anisotropy. The anisotropy parameters are the same as in Fig. 3. Lines are the splines for numerical data represented by squares and circles. Inset shows simulation geometry. 
angle $\alpha=90^{\circ}$ maximizes the outflow of the energy for the hyperbolic material.

In conclusion, we have proposed a novel phenomenon of the radiation reaction torque which can be enhanced in a metamaterial with hyperbolic dispersion. The quantum theory of the effect in an arbitrary lossy and dispersive material environment has been developed which is in full agreement with the classical quasistatic model in the case of elliptic dispersion. However, the classical description fails in the hyperbolic case, leading to unphysical, complex-valued torques. An idealized, homogeneous metamaterial shows orders of magnitude enhancement of the torque, while in a real layered hyperbolic metamaterial the effect is larger than in metamaterials with elliptic dispersion. Considering a rhodamine-like molecule in the medium with $10 \%$ anisotropy, we have estimated the expected self-induced torque (in 3D case) of several $\mathrm{pN}$. $\mathrm{nm}$, which is relevant for biological applications, where the considerable mechanical moments have the same order of magnitude. Torques play significant roles in molecular dynamics, such as DNA folding [23], and, thus, the proposed mechanism could be applied to control the torque by illuminating molecules placed within carefully engineered nanostructures [24].

This work was supported by EPSRC (UK), ERC (Project No. 321268 iPLASMM), the Ministry of Education and Science (Russia), and the Australian Research Council (Australia). P. G. acknowledges support from the Royal Society's Newton International Fellowship. This work was also supported in part by the Ministry of Education and Science of the Russian Federation (Grants No. 11.G34.31.0020, No. 14.B37.21.1649, and No. 14.B37.21.1941), the Russian Foundation for Basic Research (Grants No. 12-02-12097 and No. 12-0233034) and the Grant of President of Russian Federation (Grant No. MD-6805.2013.2).

*Corresponding author. pavel.ginzburg@kcl.ac.uk

[1] J. Kepler, Avgvstae Vindelicorvm (1619).

[2] P. Lebedew, Ann. Phys. (Berlin) 311, 433 (1901).

[3] A. Ashkin, Phys. Rev. Lett. 24, 156 (1970).
[4] A. Ashkin and J.M. Dziedzic, Science 235, 1517 (1987).

[5] M. T. Woodside, P. C. Anthony, W. M. Behnke-Parks, K. Larizadeh, D. Herschlag, and S. M. Block, Science 314, 1001 (2006).

[6] D. J. Wineland, R.E. Drullinger, and F. L. Walls, Phys. Rev. Lett. 40, 1639 (1978).

[7] K. Xiao and D. G. Grier, Phys. Rev. Lett. 104, 028302 (2010).

[8] I Bloch, Nat. Phys. 1, 23 (2005).

[9] J.I. Cirac and P. Zoller, Phys. Rev. Lett. 74, 4091 (1995).

[10] R. M. Georgevic, J. Astronautical Sci. 20, 257 (1973).

[11] L. Novotny and B. Hecht, Principles of Nano-Optics (Cambridge University Press, Cambridge, England, 2006).

[12] P. W. Milonni, The Quantum Vacuum: An Introduction to Quantum Electrodynamics (Academic, New York, 1994).

[13] M. L. Juan, M. Righini, and R. Quidant, Nat. Photonics 5, 349 (2011).

[14] V. Veselago, Sov. Phys. Usp. 10, 509 (1968).

[15] H. J. Lezec, A. K. Agrawal, M. Abashin, and K. J. Chau, Proceedings of the International Semiconductor Device Research Symposium (ISDRS), College Park, MD, 2011 (IEEE, New York, 2011); A. Dogariu, S. Sukhov, and J. Sáenz, Nat. Photonics 7, 24 (2012).

[16] N. A. R. Bhat and J.E. Sipe, Phys. Rev. A 73, 063808 (2006).

[17] J. J. Hopfield, Phys. Rev. 112, 1555 (1958); B. Huttner and S. M. Barnett, Phys. Rev. A 46, 4306 (1992).

[18] W. Vogel and D. Welsch, Quantum Optics (Wiley-VCH, Berlin, 2006).

[19] J.A. Crosse and S. Scheel, Phys. Rev. A 81, 033815 (2010).

[20] A. N. Poddubny, P. A. Belov, and Y. S. Kivshar, Phys. Rev. A 84, 023807 (2011).

[21] A. V. Kabashin, P. Evans, S. Pastkovsky, W. Hendren, G. A. Wurtz, R. Atkinson, R. Pollard, V. A. Podolskiy, and A. V. Zayats, Nat. Mater. 8, 867 (2009).

[22] H. N.S. Krishnamoorthy, Z. Jacob, E. Narimanov, I. Kretzschmar, and V.M. Menon, Science 336, 205 (2012).

[23] Z. Bryant, M. D. Stone, J. Gore, S. B. Smith, N. R. Cozzarelli, and C. Bustamante, Nature (London) 424, 338 (2003).

[24] K. C. Neuman and A. Nagy, Nat. Methods 5, 491 (2008). 